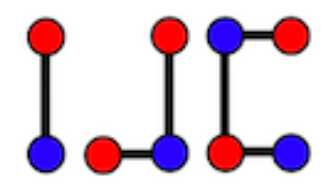

\title{
On local antimagic vertex coloring of corona products related to friendship and fan graph
}

\author{
Zein Rasyid Himami, Denny Riama Silaban* \\ Department of Mathematics, FMIPA, Universitas Indonesia, Depok, Indonesia \\ \{zein.rasyid, denny\}@sci.ui.ac.id
}

\begin{abstract}
Let $G=(V, E)$ be connected graph. A bijection $f: E \rightarrow\{1,2,3, \ldots,|E|\}$ is a local antimagic of $G$ if any adjacent vertices $u, v \in V$ satisfies $w(u) \neq w(v)$, where $w(u)=\Sigma_{e \in E(u)} f(e), E(u)$ is the set of edges incident to $u$. When vertex $u$ is assigned the color $w(u)$, we called it a local antimagic vertex coloring of $G$. A local antimagic chromatic number of $G$, denoted by $\chi_{l a}(G)$, is the minimum number of colors taken over all colorings induced by the local antimagic labeling of $G$. In this paper, we determine the local antimagic chromatic number of corona product of friendship and fan with null graph on $m$ vertices, namely, $\chi_{l a}\left(F_{n} \odot \overline{K_{m}}\right)$ and $\chi_{l a}\left(f_{(1, n)} \odot \overline{K_{m}}\right)$.

Keywords: Corona product, fan, friendship, local antimagic vertex coloring, local antimagic chromatic number Mathematics Subject Classification: 05C15, 05C76, 05C78

DOI: 10.19184/ijc.2021.5.2.7
\end{abstract}

\section{Introduction}

All graphs $G=(V, E)$ considered in this paper are simple and finite. A vertex coloring of a graph $G$ is an assignment of color to vertices of $G$ such that every two adjacent vertices have a different color. A $k$-coloring of $G$ is defined as a map $h: V \rightarrow\{1,2, \ldots, k\}$ such that $h(u) \neq h(v)$ for any adjacent vertices $u, v \in V$. The chromatic number of $G$, denoted by $\chi(G)$, is the smallest positive integer $k$ assigned to $G$.

\footnotetext{
*Corresponding author

Received: 24 July 2021, Revised: 29 October 2021, Accepted: 15 December 2021.
} 
Hartsfield and Ringel [6] introduced the principle of antimagic labeling, and then Gallian [4] surveyed the researches conducted on graph labeling and its variation, including antimagic labeling. The antimagic on a graph is defined as follows. Let $f: E \rightarrow\{1,2,3, \ldots,|E|\}$ be a bijection. The weight of vertex $u$, denoted $w(u)$, is defined as $w(u)=\Sigma_{e \in E(u)} f(e)$, where $E(u)$ is the set of edges incident to $u$. The graph $G$ is called antimagic if $w(u) \neq w(v)$, for every two vertices $u, v \in V$. Arumugam et al. [1] introduced the term of local antimagic as follows. A graph $G$ is called local antimagic if $w(u) \neq w(v)$, for any adjacent vertices $u, v \in V$. If for every distinct weight we assign distinct color, then it is called local antimagic vertex coloring. The local chromatic number of $G$, denoted by $\chi_{l a}(G)$, is the minimum number of colors taken over all colorings induced by local antimagic labeling of $G$. The local chromatic number of some graphs has been discovered, such as a tree, path, cycle, friendship, complete bipartite, an amalgamation of paths, wheel [3], kite, and cycle with two pendants [9].

Putri et al. [11] initiated a variation of local antimagic coloring named local antimagic total vertex labeling where the label is assigned to the vertices and edges of $G$. The weight of vertex $u \in$ $V, \mathrm{w}(\mathrm{u})$, is the sum of labels of all edges incident with $u$ and the label of $u$ itself. A local antimagic total chromatic number of $G$, denoted by $\chi_{l a t}(G)$, is the minimum number of colors induced by local vertex antimagic total labeling of $G$. The local antimagic total chromatic number of some graphs have been discovered such as star, a double star, banana tree, centipede, amalgamation of graphs [11] and the corona product of some graphs with $K_{2}$ [7].

If the vertices of $G$ received the smaller label in the local antimagic total labeling, then it is called the super local antimagic total. While, when the smaller labels are assigned to edges of $G$, it is called super edge local antimagic total labeling. The super local antimagic chromatic number and the super edge local antimagic chromatic number of $G$ is denoted by $\chi_{\text {slat }}(G)$ and $\chi_{\text {selat }}(G)$ respectively. The super local antimagic chromatic number of some graphs have been discovered such as fan, gear, sunflower [10], star, double star, cycle, path, cubic bipartite, wheel, amalgamation of graph, and several joint product graphs [12]. On the other hand, the super edge local antimagic total chromatic number has been discovered for path and its derivation, hedge, hedgerow, star, and an amalgamation of graphs [5].

A corona product of $H$ and $G$, denoted by $G \odot H$, is a graph obtained by taking one copy of $G$ along with $|V(G)|$ copies of $H$ and putting extra edges making the $i$-th vertex of $G$ adjacent to every vertex of the $i$-th copy of $H$ [3]. A null graph on $m$ vertices, denoted by $\overline{K_{m}}$, as a graph that has $m$ isolated vertices [2].

In this paper, we study the local antimagic chromatic number of corona products of friendship and fan with a null graph on $m$ vertices. Arumugam et al. [1] proved a sharp lower bound for any tree, and Lau et al. [8] generalized the theorem as follows.

Theorem 1.1. [8] Let $G$ be a graph having $k$ pendants. If $G$ is not $K_{2}$, the $\chi_{l a}(G) \geq k+1$ and the bound is sharp.

\section{Main Results}

\subsection{Corona Products of Friendship and Null Graphs}

A friendship graph $F_{n}$ can be constructed by joining $n$ copies of $C_{3}$ with a common vertex. Figure 1 illustrates the graph $F_{n} \odot \overline{K_{m}}$. Since $F_{1} \cong C_{3}$ and Arumugam et al. [2] already give 
$\chi_{l a}\left(C_{n} \odot \overline{K_{m}}\right)$, here we consider $F_{n} \odot \overline{K_{m}}$ for $n \geq 2$ and $m \geq 1$.

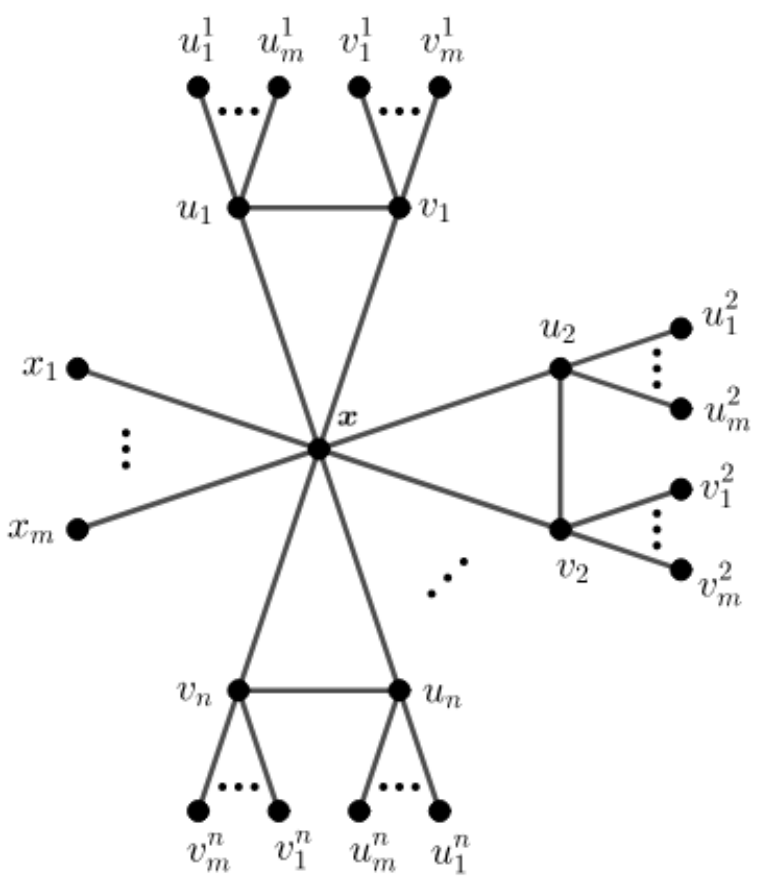

Figure 1. The graf $F_{n} \odot \overline{K_{m}}$

Theorem 2.1. Let $F_{n}$ be fan graph on $n$ cycles and $\overline{K_{m}}$ null graph on $m$ vertices. For $n \geq 2$ and $m \geq 1, \chi_{l a}\left(F_{n} \odot \overline{K_{m}}\right)=m(2 n+1)+3$.

Proof. Let $V\left(F_{n} \odot \overline{K_{m}}\right)=\left\{x, v_{i}, u_{i}, v_{j}^{i}, u_{j}^{i}, x_{j} \mid 1 \leq i \leq n\right.$ and $\left.1 \leq j \leq m\right\}$ and $E\left(F_{n} \odot \overline{K_{m}}\right)=$ $\left\{x u_{i}, x v_{i}, x x_{j}, u_{i} v_{i}, u_{i} u_{j}^{i}, v_{i} v_{j}^{i} \mid 1 \leq i \leq n\right.$ and $\left.1 \leq j \leq m\right\}$.

For the upper bound, we show that $\chi_{l a}\left(F_{n} \odot \overline{K_{m}}\right) \leq m(2 n+1)+3$. Define $f: E \rightarrow$ $\{1,2, \ldots, m(2 n+1)+3 n\}$. Label $u_{i} v_{i}, x u_{i}, x v_{i}$, and $x x_{j}$ for $1 \leq i \leq n$ and $1 \leq j \leq m$ as follows.

$$
\begin{aligned}
f\left(u_{i} v_{i}\right) & =i, \\
f\left(x u_{i}\right) & =3 n+2-2 i, \\
f\left(x v_{i}\right) & =3 n+1-2 i, \\
f\left(x x_{j}\right) & =2 m n+3 n+j .
\end{aligned}
$$

Then, to label $u_{i} u_{j}^{i}$ and $v_{i} v_{j}^{i}$ for $1 \leq i \leq n$ and $1 \leq j \leq m$, we divide into two cases according to parity of $m$.

Case 1. $m$ is odd 
For $1 \leq i \leq n$ and $1 \leq j \leq m$, label the edges as follows.

$$
\begin{aligned}
f\left(u_{i} u_{j}^{i}\right) & = \begin{cases}n(2 j+1)+i, & j \text { is odd }, \\
2 n(j+1)+1-i, & j \text { is even, }\end{cases} \\
f\left(v_{i} v_{j}^{i}\right) & = \begin{cases}2 n(j+1)+i, & j \text { is odd }, \\
n(2 j+3)+1-i, & j \text { is even. }\end{cases}
\end{aligned}
$$

The labeling $f$ is obviously a local antimagic with weights as follows.

$$
\begin{aligned}
& w\left(u_{i}\right)=m^{2} n+m n+4 n+2+\frac{3 m n+m-3 n-1}{2}, \\
& w\left(v_{i}\right)=m^{2} n+2 m n+2 n+1+\frac{3 m n+m+n-1}{2}, \\
& w(x)=4 n^{2}+n+2 m^{2} n+3 m n+\frac{m^{2}+m}{2}, \\
& w\left(x_{j}\right)=2 m n+3 n+j, \\
& w\left(u_{j}^{i}\right)= \begin{cases}n(2 j+1)+i, & j \text { is odd, } \\
2 n(j+1)+1-i, & j \text { is even, }\end{cases} \\
& w\left(v_{j}^{i}\right)= \begin{cases}2 n(j+1)+i, & j \text { is odd } \\
n(2 j+3)+1-i, & j \text { is even. }\end{cases}
\end{aligned}
$$

Case 2. $m$ is even

For $1 \leq i \leq n$ and $1 \leq j \leq m$, label the edges as follows.

$$
\begin{aligned}
& f\left(u_{i} u_{j}^{i}\right)= \begin{cases}3 n+2 i-1, & j=1, \\
n(2 j+1)+i, & j \neq 1 \text { and } j \text { is odd } \\
2 n(j+1)+1-i, & j \text { is even, }\end{cases} \\
& f\left(v_{i} v_{j}^{i}\right)= \begin{cases}3 n+2 i, & j=1, \\
2 n(j+1)+i, & j \neq 1 \text { and } j \text { is odd } \\
n(2 j+3)+1-i, & j \text { is even. }\end{cases}
\end{aligned}
$$

The labeling $f$ is obviously a local antimagic with weights as follows.

$$
\begin{aligned}
& w\left(u_{i}\right)=m^{2} n+2 m n+3 n+1+\frac{m n+m}{2}, \\
& w\left(v_{i}\right)=m^{2} n+m n+2 n+1+\frac{5 m n+m}{2}, \\
& w(x)=4 n^{2}+n+2 m^{2} n+3 m n+\frac{m^{2}+m}{2}, \\
& w\left(x_{j}\right)=2 m n+3 n+j, \\
& w\left(u_{j}^{i}\right)= \begin{array}{ll}
3 n+2 i-1, & j=1, \\
n(2 j+1)+i, & j \neq 1 \text { and } j \text { is odd, } \\
2 n(j+1)+1-i, & j \text { is even, }
\end{array} \\
& w\left(v_{j}^{i}\right)= \begin{cases}3 n+2 i, & j=1, \\
2 n(j+1)+i, & j \neq 1 \text { and } j \text { is odd }, \\
n(2 j+3)+1-i, & j \text { is even. }\end{cases}
\end{aligned}
$$

Note that for $1 \leq i \leq n, 1 \leq j \leq m$, the weights of $u_{j}^{i}, v_{j}^{i}$, and $x_{j}$ depend on $i$ and $j$ while the weight of $u_{i}, v_{i}$, and $x$ are constant. Hence, we have $2 m n+m+3$ different weights in total. 
Therefore, $\chi_{l a}\left(F_{n} \odot \overline{K_{m}}\right) \leq m(2 n+1)+3$.

For the lower bound, we show that $\chi_{l a}\left(F_{n} \odot \overline{K_{m}}\right) \geq m(2 n+1)+3$. Since $F_{n} \odot \overline{K_{m}}$ has $2 m n+m$ pendants, by using Theorem 1.1, we have $\chi_{l a}\left(F_{n} \odot \overline{K_{m}}\right) \geq 2 m n+m+1$. Suppose $\chi_{l a}\left(F_{n} \odot \overline{K_{m}}\right) \geq 2 m n+m+1$. Then, $w(x)$ will equal to either $w\left(u_{j}^{i}\right)$ or $w\left(v_{j}^{i}\right)$ for some $i$ and $j$. Since $d(x)=2 n+m$, we obtain $w(x) \geq \sum_{k=1}^{2 n+m} k=\frac{(2 n+m)(2 n+m+1)}{2}$, where $d(x)$ is degree of vertex $x$. On the other hand, the weights of either $w\left(u_{j}^{i}\right)$ or $w\left(v_{j}^{i}\right) \leq|E|=2 m n+m+3 n$ which implies $w(x) \geq \frac{(2 n+m)(2 n+m+1)}{2}=2 n^{2}+2 m n+n+\frac{m^{2}+m}{2} \geq 2 m n+(2 n+1) n+m>2 m n+3 n+m$. It is a contradiction. Therefore, the color of $w(x)$ must be different from all pendants and now we have extended the lower bound to $\chi_{l a}\left(F_{n} \odot \overline{K_{m}}\right) \geq 2 m n+m+2$.

Suppose $\chi_{l a} \geq 2 m n+m+2$. Then, either $w\left(u_{i}\right)=w\left(v_{j}^{i}\right)$ or $w\left(v_{i}\right)=w\left(u_{j}^{i}\right)$ must be satisfy for some $j$. Suppose $w\left(u_{i}\right)=w\left(v_{j}^{i}\right)$. Notice that $w\left(u_{i}\right) \geq \frac{\sum_{k=1}^{2 n+m n} k}{n}=\frac{(2 n+m n)(2 n+m n+1)}{2 n}$, while $w\left(v_{j}^{i}\right) \leq 2 m n+m+3 n$. It is not hard to verify that $\frac{(2 n+m n)(2 n+m n+1)}{2 n}=2 m n+\left(\frac{m n+1}{2}\right) m+4 n+2>$ $2 m n+m+3 n$ for $n \geq 2$ and $m \geq 1$. It is a contradiction since $w\left(v_{j}^{i}\right)<w\left(u_{i}\right)$. We can construct the same argument to show a contradiction for the case $w\left(u_{i}\right)=w\left(x_{j}\right)$ or $w\left(v_{i}\right)=w\left(x_{j}\right)$ for some $j$. Therefore, the color of $w\left(v_{i}\right)$ must be different from all pendants and now we have extended the lower bound to $\chi_{l a}\left(F_{n} \odot \overline{K_{m}}\right) \geq 2 m n+m+3$.

Since both inequalities $\chi_{l a}\left(F_{n} \odot \overline{K_{m}}\right) \leq 2 m n+m+3$ and $\chi_{l a}\left(F_{n} \odot \overline{K_{m}}\right) \geq 2 m n+m+3$ hold, then $\chi_{l a}\left(F_{n} \odot \overline{K_{m}}\right)=2 m n+m+3$.

We give the local antimagic vertex coloring for $F_{5} \odot \overline{K_{3}}$ with $\chi_{l a}\left(F_{5} \odot \overline{K_{3}}\right)=36$ in Figure 2 .

\subsection{Corona Products of Fan and Null Graphs}

A fan graph $f_{(1, n)}$ is defined as the graph $K_{1}+P_{n}$ where $K_{1}$ is the null graph on one vertex and $P_{n}$ is the path graph on $n$ vertices. Figure 3 illustrates the graph $f_{(1, n)} \odot \overline{K_{m}}$. Since $f_{(1,2)} \cong C_{3}$ and Arumugam et al. [2] already give $\chi_{l a}\left(C_{n} \odot \overline{K_{m}}\right)$, here we consider $f_{(1, n)} \odot \overline{K_{m}}$ for $n \geq 3$ and $m \geq 1$.

Theorem 2.2. Let $f_{(1, n)}$ be friendship of $n+1$ vertices and $\overline{K_{m}}$ null graph on $m$ vertices. For $n \geq 3$ and $m \geq 1, \chi_{l a}\left(f_{(1, n)} \odot \overline{K_{m}}\right)=m(n+1)+3$.

Proof. Let $V\left(f_{(1, n)} \odot \overline{K_{m}}\right)=\left\{x, v_{i}, v_{j}^{i}, x_{j} \mid 1 \leq i \leq n\right.$ and $\left.1 \leq j \leq m\right\}$ and $E\left(f_{(1, n)} \odot \overline{K_{m}}\right)=$ $\left\{x x_{j}, x v_{i}, v_{i} v_{i+1}, v_{i} v_{j}^{i} \mid 1 \leq i \leq n\right.$ and $\left.1 \leq j \leq m\right\}$.

For the upper bound, we show that $\chi_{l a}\left(f_{(1, n)} \odot \overline{K_{m}}\right) \leq m(n+1)+3$. Define $f: E \rightarrow$ $\{1,2, \ldots, m(n+1)+2 n-1\}$. We divide into two cases depend on the parity of $n$.

Case 1. $n$ is odd 


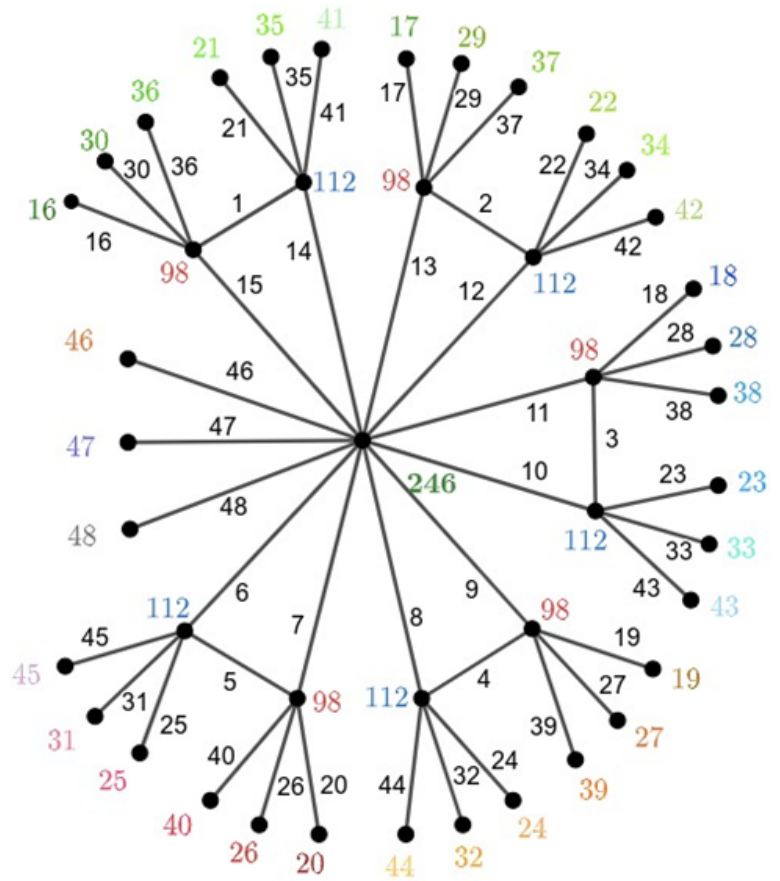

Figure 2. The local antimagic vertex coloring of $F_{5} \odot \overline{K_{3}}$

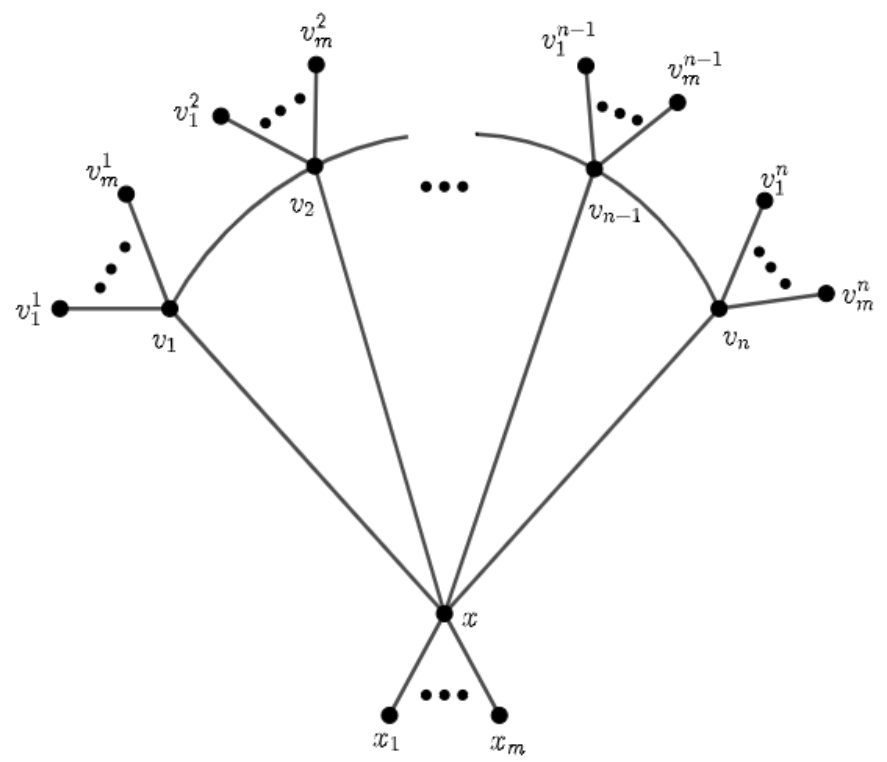

Figure 3. The graph $f_{(1, n)} \odot \overline{K_{m}}$ 
Label the edges $v_{i} v_{i+1}, x v_{i}$, and $x x_{j}$ for $1 \leq i \leq n$ and $1 \leq j \leq m$ as follows.

$$
\begin{aligned}
f\left(v_{i} v_{i+1}\right) & = \begin{cases}\frac{i+1}{2}, & i \text { is odd }, \\
n-1-\frac{i-2}{2}, & i \text { is even, }\end{cases} \\
f\left(x v_{i}\right) & = \begin{cases}2 n-1, & i=1, \\
n+\frac{i-3}{2}, & i \neq 1 \text { and } i \text { is odd }, \\
n-1+\frac{n-1+i}{2}, & i \text { is even, }\end{cases} \\
f\left(x x_{j}\right) & =m n+m+2 n-j .
\end{aligned}
$$

Then, to label $v_{i} v_{j}^{i}$ for $1 \leq i \leq n$ and $1 \leq j \leq m$, we divide into two subcases according to parity of $m$.

Subcase 1. $m$ is odd

Label $v_{i} v_{j}^{i}$ for $1 \leq i \leq n$ and $1 \leq j \leq m$ as follows.

$$
\begin{aligned}
& f\left(v_{i} v_{1}^{i}\right)= \begin{cases}2 n+1+\frac{n-3}{2}, & i=n, \\
2 n+\frac{n-i-2}{2}, & i \neq n \text { and } i \text { is odd }, \\
3 n-1-\frac{i-2}{2}, & i \text { is even, }\end{cases} \\
& f\left(v_{i} v_{j}^{i}\right)= \begin{cases}(j+1) n-1+i, & j \neq 1 \text { and } j \text { is odd }, \\
(j+2) n-i, & j \text { is even. }\end{cases}
\end{aligned}
$$

The labeling $f$ is obviously a local antimagic with weights

$$
\begin{aligned}
w\left(v_{i}\right)= & \begin{cases}2 m n+2 n-1+\frac{m^{2}-m}{2^{2}}, & i \text { is odd }, \\
2 m n+3 n-1+\frac{m^{2}-m}{2}, & i \text { is even. }\end{cases} \\
w(x)= & m^{2} n+2 m n+\frac{m^{2}-m+3 n^{2}-n}{2}, \\
w\left(x_{j}\right)= & m n+m+2 n-j, \\
w\left(v_{j}^{i}\right)= & \begin{cases}2 n+1+\frac{n-3}{2}, & j=1 ; i=n, \\
2 n+\frac{n-i-2}{2}, & j=1 ; i \neq n \text { and } i \text { is odd }, \\
3 n-1-\frac{i-2}{2}, & j=1 ; i \text { is even, } \\
(j+1) n-1+i, & j \neq 1 \text { and } j \text { is odd }, \\
(j+2) n-i, & j \text { is even. }\end{cases}
\end{aligned}
$$

Subcase 2. $m$ is even

Label $v_{i} v_{j}^{i}$ for $1 \leq i \leq n$ and $1 \leq j \leq m$ as follows.

$$
\begin{aligned}
& f\left(v_{i} v_{1}^{i}\right)= \begin{cases}3 n-1, & i=n, \\
3 n-2-i, & i \neq n \text { and } i \text { is odd }, \\
3 n-i, & i \text { is even, }\end{cases} \\
& f\left(v_{i} v_{2}^{i}\right)= \begin{cases}3 n, & i=n, \\
3 n+\frac{i+1}{2}, & i \neq n \text { and } i \text { is odd }, \\
3 n+\frac{n-1+i}{2}, & i \text { is even, }\end{cases} \\
& f\left(v_{i} v_{j}^{i}\right)= \begin{cases}(j+1) n-1+i, & j \neq 1 \text { and } j \text { is odd }, \\
(j+2) n-i, & j \neq 2 \text { and is even. }\end{cases}
\end{aligned}
$$


The labeling $f$ is obviously a local antimagic with weights

$$
\begin{aligned}
& w\left(v_{i}\right)= \begin{cases}2 m n+2 n+1+\frac{m^{2} n-m}{2}, & i \text { is odd, } \\
2 m n+3 n-1+\frac{m^{2} n-m}{2}, & i \text { is even, }\end{cases} \\
& w(x)=m^{2} n+2 m n+\frac{m^{2}-m+3 n^{2}-n}{2}, \\
& w\left(x_{j}\right)=m n+m+2 n-j, \\
& w\left(v_{j}^{i}\right)= \begin{cases}3 n-1, & j=1 ; i=n, \\
3 n-2-i, & j=1 ; i \neq n \text { and } i \text { is odd, } \\
3 n-i, & j=1 ; i \text { is even, } \\
3 n, & j=2 ; i=n, \\
3 n+\frac{i+1}{2}, & j=2 ; i \neq n \text { and } i \text { is odd, } \\
3 n+\frac{n-1+i}{2}, & j=2 ; i \text { is even, } \\
(j+1) n-1+i, & j \neq 1 \text { and } j \text { is odd, } \\
(j+2) n-i, & j \neq 2 \text { and is even. }\end{cases}
\end{aligned}
$$

Case 2. $n$ is even

Label the edges $\left\{v_{i} v_{i+1}, x v_{i}\right.$, and $\left.x x_{j}\right\} 1 \leq i \leq n$ and $1 \leq j \leq m$ as follows.

$$
\begin{aligned}
f\left(v_{i} v_{i+1}\right) & = \begin{cases}\frac{i+1}{2}, & i \text { is odd }, \\
n-1-\frac{i-2}{2}, & i \text { is even, }\end{cases} \\
f\left(x v_{i}\right) & = \begin{cases}2 n-1, & i=1, \\
2 n-2, & i=n, \\
n+\frac{i-3}{2}, & i \neq 1 \text { and } i \text { is odd } \\
n-2+\frac{n+i}{2}, & i \neq n \text { and } i \text { is even, }\end{cases} \\
f\left(x x_{j}\right) & =m n+m+2 n-j .
\end{aligned}
$$

Then, to label $v_{i} v_{j}^{i}$ for $1 \leq i \leq n$ and $1 \leq j \leq m$, we divide into two subcases according to parity of $m$.

Subcase 1. $m$ is odd

Label $v_{i} v_{j}^{i}$ for $1 \leq i \leq n$ and $1 \leq j \leq m$ as follows.

$$
\begin{aligned}
& f\left(v_{i} v_{1}^{i}\right)= \begin{cases}3 n-1, & i=n, \\
3 n-1-\frac{i}{2}, & i \neq n \text { and } i \text { is even }, \\
2 n+\frac{n-1-1}{2}, & i \text { is odd },\end{cases} \\
& f\left(v_{i} v_{j}^{i}\right)= \begin{cases}(j+1) n-1+i, & j \neq 1 \text { and } j \text { is odd }, \\
(j+2) n-i, & j \text { is even. }\end{cases}
\end{aligned}
$$


The labeling $f$ is obviously a local antimagic with weights

$$
\begin{aligned}
w\left(v_{i}\right)= & \begin{cases}2 m n+2 n-1+\frac{m^{2} n-m+1}{2}, & i \text { is odd, } \\
2 m n+3 n-3+\frac{m^{2} n-m+1}{2}, & i \text { is even, }\end{cases} \\
w(x)= & m^{2} n+2 m n+\frac{m^{2}-m+3 n^{2}-n}{2}, \\
w\left(x_{j}\right)= & m n+m+2 n-j, \\
w\left(v_{j}^{i}\right)= & \begin{cases}3 n-1, & j=1 ; i=n, \\
3 n-1-\frac{i}{2}, & j=1 ; i \neq n \text { and } i \text { is even, } \\
2 n+\frac{n-i-1}{2}, & j=1 ; i \text { is odd, } \\
(j+1) n-1+i, & j \neq 1 \text { and } j \text { is odd }, \\
(j+2) n-i, & j \text { is even. }\end{cases}
\end{aligned}
$$

Subcase 2. $m$ is even

Label $v_{i} v_{j}^{i}$ for $1 \leq i \leq n$ and $1 \leq j \leq m$ as follows

$$
\begin{aligned}
& f\left(v_{i} v_{1}^{i}\right)= \begin{cases}3 n-1, \quad i=n, \\
3 n-1-i, \quad i \neq n,\end{cases} \\
& f\left(v_{i} v_{2}^{i}\right)= \begin{cases}3 n+1+\frac{n-2}{2}, & i=n, \\
3 n+1+\frac{n+i-2}{2}, & i \neq n \text { and } i \text { is even, } \\
3 n+\frac{i-1}{2}, & i \text { is odd, }\end{cases} \\
& f\left(v_{i} v_{j}^{i}\right)= \begin{cases}(j+1) n-1+i, & j \neq 1 \text { and } j \text { is odd }, \\
(j+2) n-i, & j \neq 2 \text { and } j \text { is even. }\end{cases}
\end{aligned}
$$

The labeling $f$ is obviously a local antimagic with weights

$$
\begin{aligned}
w\left(v_{i}\right)= & \begin{cases}2 m n+2 n-1+\frac{m^{2} n-m}{2}, & i \text { is odd, } \\
2 m n+3 n-2+\frac{m^{2} n-m}{2}, & i \text { is even, }\end{cases} \\
w(x)= & m^{2} n+2 m n+\frac{m^{2}-m+3 n^{2}-n}{2},
\end{aligned} \begin{aligned}
w\left(x_{j}\right)= & m n+m+2 n-j, \\
w\left(v_{j}^{i}\right)= & \begin{cases}3 n-1, & j=1 ; i=n, \\
3 n-1-i, & j=1 ; i \neq n, \\
3 n+1+\frac{n-2}{2}, & j=2 ; i=n, \\
3 n+1+\frac{n+i-2}{2}, & j=2 ; i \neq n \text { and } i \text { is even, } \\
3 n+\frac{i-1}{2}, & j=2 ; i \text { is odd, } \\
(j+1) n-1+i, & j \neq 1 \text { and } j \text { is odd, } \\
(j+2) n-i, & j \neq 2 \text { and } j \text { is even. }\end{cases}
\end{aligned}
$$

Since for $1 \leq i \leq n$ and $1 \leq j \leq m$, the weights of $v_{j}^{i}$ and $x x_{j}$ depend on $i$ and $j$, while the weight of $v_{i}$ and $x$ are constant, we have $m n+m+3$ different weights in total. Therefore, $\chi_{l a}\left(f_{(1, n)} \odot \overline{K_{m}}\right) \leq m n+m+3$.

For the lower bound, we show that $\chi_{l a}\left(f_{(1, n)} \odot \overline{K_{m}}\right) \geq m n+m+3$. Since $f_{(1, n)} \odot \overline{K_{m}}$ has $m n+m$ pendants, by using Theorem 1.1, we have $\chi_{l a}\left(f_{(1, n)} \odot \overline{K_{m}}\right) \geq m n+m+1$. Suppose 
$\chi_{l a}\left(f_{(1, n)} \odot \overline{K_{m}}\right) \geq m n+m+1$. Then, $w(x)$ must equal to $w\left(v_{j}^{i}\right)$ for some $i$ and $j$. Note that $w(x) \geq \sum_{k=1}^{m+n} k=\frac{(m+n)(m+n+1)}{2}$, while $w\left(v_{j}^{i}\right) \leq m n+m+2 n-1$ for any $i$ and $j$. It is not hard to verify that $\frac{(m+n)(m+n+1)}{2}=m n+\left(\frac{m+1}{2}\right) m+\left(\frac{n+1}{2}\right) n>m n+m+2 n-1$, if $n \geq 3$. Hence, we get a contradiction. Therefore, $\chi_{l a}\left(f_{(1, n)} \odot \overline{K_{m}}\right) \geq m n+m+2$.

Now, suppose $\chi_{l a}\left(f_{(1, n)} \odot \overline{K_{m}}\right) \geq m n+m+2$. Since $w(x)$ is unique, there must be at least $\left\lfloor\frac{n}{2}\right\rfloor$ pairs of vertices such that $w\left(v_{i}\right)=w\left(v_{j}^{i}\right)$ for some $i$ and $j$. We will show that it impossible by considering the parity of $n$. First, if $n$ is even, $w\left(v_{i}\right) \geq \frac{\frac{\frac{m n+3 n+2}{2} k}{k} k}{\frac{n}{2}}=\frac{\left(\frac{m n+3 n+2}{2}\right)\left(\frac{m n+3 n+4}{2}\right)}{n}$ for all $i$, while $w\left(v_{j}^{i}\right) \leq(n+1) m+2 n-1$ for all $i$ and $j$. It is not hard to verify that $\frac{\left(\frac{m n+3 n+2}{2}\right)\left(\frac{m n+3 n+4}{2}\right)}{n}=$

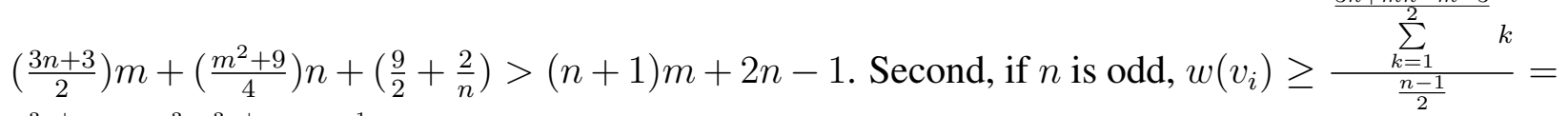
$\frac{\left(\frac{3 n+m n-m-3}{2}\right)\left(\frac{3 n+m n-m-1}{2}\right)}{(n-1)}$ for all $i$, while $w\left(v_{j}^{i}\right) \leq(n+1) m+2 n-1$ for all $i$ and $j$. It is not hard to verify that $\frac{\left(\frac{3 n+m n-m-3}{2}\right)\left(\frac{3 n+m n-m-1}{2}\right)}{(n-1)}=\frac{m^{2} n^{2}-2 m^{2} n+6 m n^{2}-10 m n+9 n^{2}+m^{2}+4 m-12 n+3}{4 n-4}=(1+$ $\left.\frac{2 n-2 m-6}{4 n-4}\right) m n+\left(\frac{m n^{2}+m+4}{4 n-4}\right) m+\left(2+\frac{n-4}{4 n-4}\right) n+\frac{3}{4 n-4}>m n+m+2 n-1$. We have a contradiction. We can construct the same argument to show a contradiction for the case $w\left(v_{i}\right)=w\left(x_{j}\right)$ for some $j$. Therefore, $\chi_{l a}\left(f_{(1, n)} \odot \overline{K_{m}}\right) \geq m n+m+3$.

Since both $\chi_{l a}\left(f_{(1, n)} \odot \overline{K_{m}}\right) \leq m n+m+3$ and $\chi_{l a}\left(f_{(1, n)} \odot \overline{K_{m}}\right) \geq m n+m+3$ hold, then $\chi_{l a}\left(f_{(1, n)} \odot \overline{K_{m}}\right)=m n+m+3$.

We give the local antimagic vertex coloring for $f_{(1,6)} \odot \overline{K_{3}}$ with $\chi_{l a}\left(f_{(1,6)} \odot \overline{K_{3}}\right)=24$ in Figure 4 .

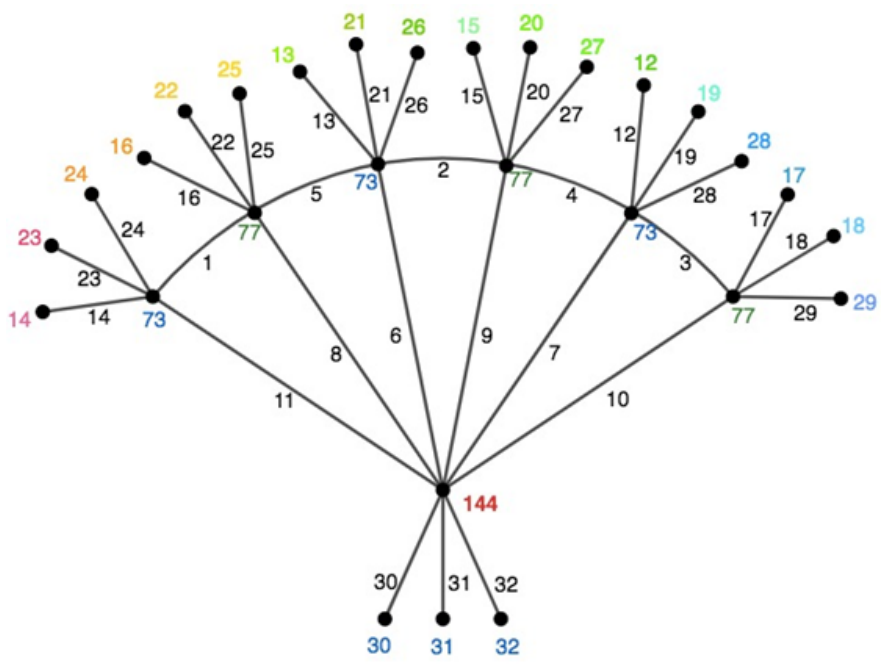

Figure 4. The local antimagic vertex coloring of $f_{(1,6)} \odot \overline{K_{3}}$ 


\section{Conclusion}

We summarise the results in Table 1.

Table 1. Summary

\begin{tabular}{llll}
\hline Corona Products of & Notation & $\chi_{l a}$ & Condition \\
\hline Friendship with Null graph on $m$ vertices & $F_{n} \odot \overline{K_{m}}$ & $m(2 n+1)+3$ & $n \geq 2$ and $m \geq 1$ \\
Fan with Null graph on $m$ vertices & $f_{(1, n)} \odot \overline{K_{m}}$ & $m(n+1)+3$ & $n \geq 3$ and $m \geq 1$ \\
\hline
\end{tabular}

\section{References}

[1] S. Arumugam, K. Premalatha, M. Bača, and A. Semaničová-Feňovčíková, Local antimagic vertex coloring of a graph, Graphs and Combinatorics. 33 (2017), 275-285. doi: 10.1007/s00373-017-1758-7.

[2] S. Arumugam, Yi-Chun Lee, K. Premalatha, and Tao-Ming Wang, On local antimagic vertex coloring for corona products of graph (2018), ArXiv:1808.04956v1.

[3] R. Frucht and F. Harary. On the corona of two graphs, Aequationes Math, 4 (1970), 322-325.

[4] J.A. Gallian, A dynamic survey of graph labeling, The Electronic Journal of Combinatorics, (2019), \#DS6.

[5] F.F. Hadiputra, D.R. Silaban, and T.K. Maryati, Super local edge anti-magic total coloring of paths and its derivation, Indonesian Journal of Combinatorics, 3 (2019), 126-139, doi: 10.19184/ijc.2019.3.2.6.

[6] N. Hartsfield and G. Ringel. Pearls in Graph Theory, Academic Press, (1990).

[7] E.Y. Kurniawati, I.H. Agustin, Dafik, and Marsidi, On the vertex local antimagic total labeling chromatic number of $G \odot K_{2}$, Journal of Physics: Conference Series, 1211 (2019), doi: 10.1088/1742-6596/1008/1/012035.

[8] Gee-Choon Lau, Wai-Chee Shiu, and Ho-Kuen Ng, On local antimagic chromatic number of graphs with cut-vertices, (2018), ArXiv:1805.04801v5.

[9] N.H. Nazula, S. Slamin, and D. Dafik, Local antimagic vertex coloring of uncyclic graphs, Indonesian Journal of Combinatorics, 2 (2018), 30-34, doi: 10.19184/ijc.2018.2.1.4.

[10] S.A. Pratama, S. Setiawani, and Slamin, Local super antimagic total vertex coloring of some wheel related graphs, Journal of Physics: Conference Series, 1538 (2020), doi: 10.1088/1742-6596/1538/1/012014. 
[11] D.F. Putri, Dafik, I.H. Agustin, and R. Alfarisi, On the local vertex antimagic total coloring of some families tree, Journal of Physics: Conference Series, 1008 (2018), doi: 10.1088/17426596/1008/1/012035.

[12] Slamin, N.O. Adiwijaya, M.A. Hasan, Dafik, and K. Wijaya, Local super antimagic total labeling for vertex coloring of graphs, Symmetry, 12 (2020), doi: 10.3390/sym12111843. 